\title{
REFLECTANCE SPECTROSCOPY CHARACTERIZATION OF SPACE DEBRIS
}

\author{
A. Vananti ${ }^{{ }^{*}}$, T. Schildknecht ${ }^{1}$, H. Krag $^{2}$ \\ ${ }^{1}$ Astronomical Institute, University of Bern, CH-3012 Bern, Switzerland \\ ${ }^{2}$ ESA ESOC, Robert-Bosch-Strasse 5, 64293 Darmstadt, Germany
}

Keywords: Reflectance spectroscopy, Color photometry, Space debris, Geostationary satellites

\begin{abstract}
When characterizing the space debris environment one important point is the identification of the physical properties of the space debris objects. Reflectance spectroscopy is a possible technique to study the surface material of these objects with observations from the ground. In this work the results collected from spectroscopy observations of space debris in orbit are presented. The observations were performed at the 1-meter ESA Space Debris Telescope (ESASDT) in Tenerife equipped with a spectrograph in the 450-960 nm wavelength range. A preliminary classification using three different categories purely based on the shape and appearance of the spectra was proposed. Two debris objects with high AMR could be successfully associated with materials analyzed in the laboratory. The results show that the two debris objects are probably pieces of Kapton MLI with 'gold' and 'silver' coating, respectively. Color indices were extracted from the spectroscopic measurements. For high AMR objects the colors seem to be consistent with the proposed classification in three categories. The B-R and R-I laboratory measurements of 'gold' and 'silver' MLI taken from the literature are comparable with the obtained results.
\end{abstract}

\section{INTRODUCTION}

For the study of the space debris environment, especially in higher orbits, optical observations using groundbased optical telescopes are mostly employed. Surveys for space debris allow the discovery of new objects, the subsequent determination and maintenance of their orbits, and in some case the analysis of their physical properties (Schildknecht, 2007). From the optical observations the astrometric positions and the brightness (magnitude) of the objects are extracted. The astrometric positions are used to determine and maintain the orbits of the objects. When determining the orbit also the area-to-mass ratio (AMR) can be estimated (Schildknecht et al., 2008a). From the measured magnitudes it is possible to derive an estimate of the size of the object. Nonetheless, this conversion from magnitudes to physical sizes implies the availability of data about the surface properties and the shapes of the objects like, e.g., albedo, color, specular and diffuse reflection characteristics. The variation of the magnitude as a function of the time, also called light curve, gives information about the object's shape, its tumbling rate or attitude motion (Santoni et al., 2013; Yanagisawa and Kurosaki, 2012). Light curves of geostationary (GEO) objects exhibit a variety of periodic patterns with time scales in the range from fractions of second to several minutes (Schildknecht et al., 2008b). Color photometry observations of objects in GEO-like orbits (Cardona et al., 2016; Zhao et al., 2016) could provide information on the surface material of the objects. The difficulty in this case is the color determination when the brightness changes of the objects are very fast and for equipment reasons it is not possible to acquire multiple colors measurements simultaneously.

The advantage of reflectance spectrometry is the simultaneous measurement of the brightness of the object over a wide wavelength range. The spectra show the reflection properties of a material illuminated by the Sun and the reflected intensity is dependent on wavelength. Several studies have been carried out on reflectance spectroscopy of orbiting objects (Jorgensen et al., 2004; Seitzer et al., 2012; Bédard and Wade, 2017). The analysis of rocket bodies in low Earth orbits (LEO) and GEO orbits through spectral data were reported already in 2001 (Nishimoto et al., 2001). In some articles the spectral data are compared with laboratory measurements of the same object or part of a spacecraft. Sometimes it is possible to determine particular spectral features related to certain materials as described in the work of Abercromby et al. (2007). Another interesting outcome of these investigations is that the objects exposed to the space environment for long time reveal space weathering or ageing effects. In

\footnotetext{
${ }^{*}$ Corresponding author, E-mail address: alessandro.vananti@aiub.unibe.ch
} 
those spectra a so-called "reddening" can be noticed, i.e. an increase of the slope of the reflectance with growing wavelength (Abercromby et al., 2005).

A project initiated by ESA started in 2008 with the goal to identify and assess technologies to obtain spectroscopic measurements of objects in GEO. In the project it should be analyzed if the physical information derived from the measurements is appropriate to classify objects in the frame of a European Space Surveillance program. In the course of the project, first reflectance data of small-size debris objects at high altitude were acquired. In particular observations of high AMR objects in GEO orbits were performed. Regarding this family of AMR objects, it is supposed that the latter could be fragments of light surface materials of spacecraft, like solar cells or multi-layer insulation (MLI) foils. A comparison of the observed reflectance spectra of high AMR objects with spectra of spacecraft components measured in the laboratory may allow identifying the nature and provenance of the high AMR population.

In this work the results from the spectroscopic observation campaigns performed with the 1-meter ESA Space Debris Telescope (ESASDT) in Tenerife are presented. Subsequently the spectra are compared with spectroscopic measurements in the laboratory of selected material samples, while the derived color indices are compared with values taken from the literature.

\section{EXPERIMENTAL SETUP}

The observation were conducted at the 1-meter ESASDT telescope using a spectrograph with low-resolution placed at the Ritchey-Chrétien focus. The dispersive element of this spectrograph is a combination of grating and prism, a so-called 'grism'. The prism is necessary to deviate the aimed wavelength range (order) into the optical axis. There were three grisms with different resolutions available. The choice of the grism was imposed by the low signal-to-noise values, since the target objects were estimated to be fainter than magnitude 12, and even in the range of magnitude 16 to 17 in case of high AMR objects. In general for observation of stars these magnitudes would simply involve long exposures of several tens of minutes. However, for moving objects the time available for the exposure is shorter and is mainly constrained by the density of the stellar background, at magnitudes usually brighter than the science object. Thus the measurement is corrupted whenever the object crosses a star. Due to the existing constraints an exposure time of 4 minutes and the grism with the lowest dispersion were chosen to aim at the maximal signal-to-noise ratio per pixel. The latter grism has a nominal central wavelength of $510 \mathrm{~nm}$ with a resolution of $0.4 \mathrm{~nm}$ per pixel in the range from 450 to $960 \mathrm{~nm}$. The dispersion band is relatively broad and the dispersion of the second order overlaps the same spatial area. For this reason a supplementary order separation filter is necessary for wavelengths larger than $550 \mathrm{~nm}$ and a glass filter OG515 with a cut-off position of $515 \mathrm{~nm}$ was utilized. The spectrograph is equipped with a 5 arcsec slit, an Osram He/10 spectral lamp for wavelength calibrations, and a camera with a backside illuminated CCD of 2048 x 2048 pixels and pixel size of $13.5 \times 13.5 \mu \mathrm{m}^{2}$. An autoguider system is usually available for the telescope observation and ideally it should be used with the spectrograph. The autoguider deviates the light of a selected star into a separate high-speed camera via an off-axis pickup mirror. Unfortunately this solution cannot be applied for objects with large angular velocities w.r.t. the stars. Therefore the telescope was operated in the 'open loop' tracking mode. It is known that significant tracking errors accumulate within short time caused by restrictions in the mount model. In fact test observations reveal tracking drifts around few arcseconds per minute. Consequently, even assuming perfect ephemerides, an object would therefore drift outside the largest slit within few minutes. Since there is no way to check the slit and the precise position of the object in the slit during the exposure, the largest available slit, about 6 arcsec wide, was selected. The whole focal plane component of the spectrograph can be rotated w.r.t. the telescope optical axis thanks to an extra rotation device. The latter is conceived to place the slit parallel to the atmospheric dispersion direction, i.e. perpendicular to the horizon, to avoid cutting of the dispersed object image by the slit. Nonetheless, since the rotation device can only be steered manually it was decided to use only one orientation during the whole night.

In addition to the observations at the ESASDT telescope, laboratory spectroscopic measurements were conducted at the Astronomical Institute of the University of Bern (AIUB) with a general-purpose Ocean Optics USB2000+ spectrometer.

\section{SPECTROSCOPIC DATA REDUCTION}




\section{Reduction to reflectance spectra}

For the reduction of the measured spectra a standard procedure was applied as e.g. described in (Wagner, 1992). As tool, the slit spectroscopy module of the MIDAS software (Banse et al., 1988) was selected for the following reduction steps:

- elimination of cosmic ray events on the acquired frames

- subtraction of sky background

- binning of the two-dimensional frame into a one-dimensional spectrum

- calibration of the dispersion scale based on observations of the spectrum of the calibration lamp

Subsequently observations of the same object are stacked together and the resulting spectra are normalized to unity at an arbitrarily chosen wavelength value of $550 \mathrm{~nm}$. After these reduction steps the spectra are not corrected yet for the wavelength-dependent atmospheric extinction and the response function of the spectrograph. For the next step, to obtain reflectance intensities, the spectrum of the illuminating source, namely the Sun, has to be taken into account. For technical reasons, instead of the Sun, for each object a solar analog (with a spectrum very similar to the solar one) close to the object position was observed and used for calibration. The following solar analogs were selected: SA 93-101, SA 98-978, SA 102-1081, SA 107-684, SA 107-998, SA 110-361, SA 112-1333, SA 115-271 (Landolt, 1973, 1992), Hyades 64, and 16 Cyg B (Hardorp, 1978).

Two procedures were adopted for the extinction correction: nightly extinction data resulting from the observations, or correction using mean extinction values. Extracting the extinction function from the campaign measurements yields almost real time conditions but it may lead to large errors in the extinction correction. In order to compute the extinction curve of one night, spectra of solar analogs measured at different air mass values were considered. Fig. 1 displays four spectra of a solar analog star at different air masses. The effect of the atmospheric extinction considerably modifies the shape of the observed spectra. The coefficients are calculated fitting the measured intensities at different air mass in the whole wavelength range. If only few measured points per fit are available the errors can be in the order of $0.1 \mathrm{mag} /$ airmass or higher.

Referring to standard curves has the disadvantage that the calibration measurements, taken on several nights with photometric sky conditions during the year from a given observatory, do not correspond to the real conditions of the spectroscopy campaign night. In general, the extinction can vary from night to night, and even within the same night. It also depends on the period of the year and of course on the observation site. The variance in the standard extinction data of the Teide observatory (Kidger et al., 2003) is close to the 0.1 mag/airmass mentioned above. However, this approach has the advantage that the extinction coefficients are consistent each other and in first approximation the difference from the real extinction is a constant factor for a given night. For this reason and since in general not enough calibration measurements were taken, most of the spectra were calibrated using the mean extinction coefficients of the Teide observatory.

The extinction corrected object spectra were divided by the solar analog spectra resulting in intensity-calibrated reflectance spectra. This computation is not only necessary to remove the solar spectrum dependence, but also implicitly removes the dependence from the spectrograph response function, since the latter appears in both measurements. The observed object spectrum, a solar analog spectrum, and the resulting intensity-calibrated reflectance spectrum of the object are displayed in Fig. 2. The final reduction step is a smoothing of the spectra with a low-pass filter to eliminate background noise and fringes, which originate from the interference of light, subject to multiple reflections between upper and lower CCD surface. This last step is justified by the fact that one is interested only in the spectral energy distribution and not in the single spectral lines.

The error in the reflectance can be roughly estimated considering the extinction uncertainty. Other error sources should be taken into account for a more detailed estimate. In Bédard and Wade (2017) the error introduced by using different solar analogs is reported. Given in Fig. 1 the relative difference in the reflectance related to a change of almost 1 air mass, the relative reflectance error due to the above mentioned 0.1 mag/airmass can be derived. In the following the reflectance error at $450 \mathrm{~nm}$ and $800 \mathrm{~nm}$ will be indicated as $\sigma_{450}$ and $\sigma_{800}$ respectively. 


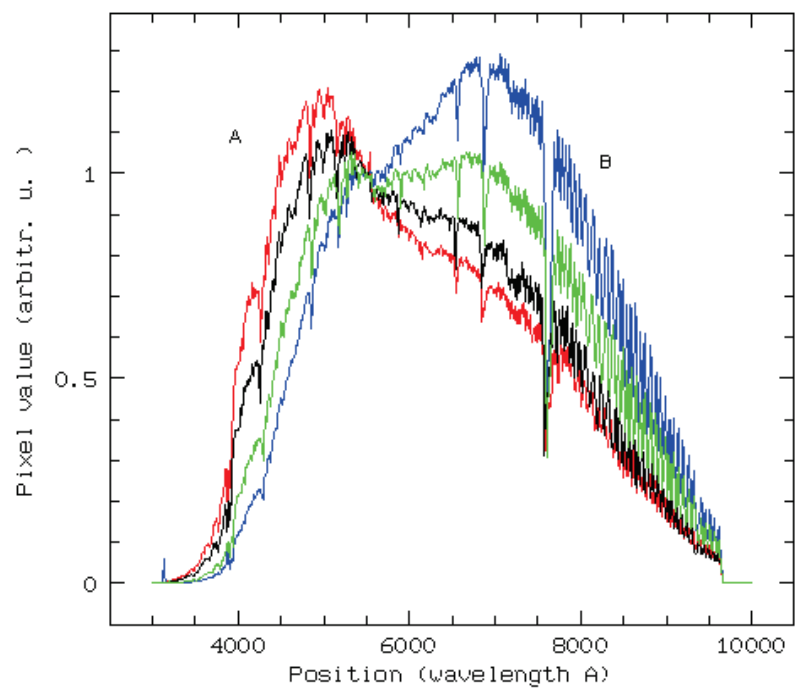

Fig. 1. Normalized spectra of solar analog stars at different air masses. The red, black, green, and blue curves are spectra obtained at 1.01, 1.13, 1.4 and 1.94 air mass, respectively.

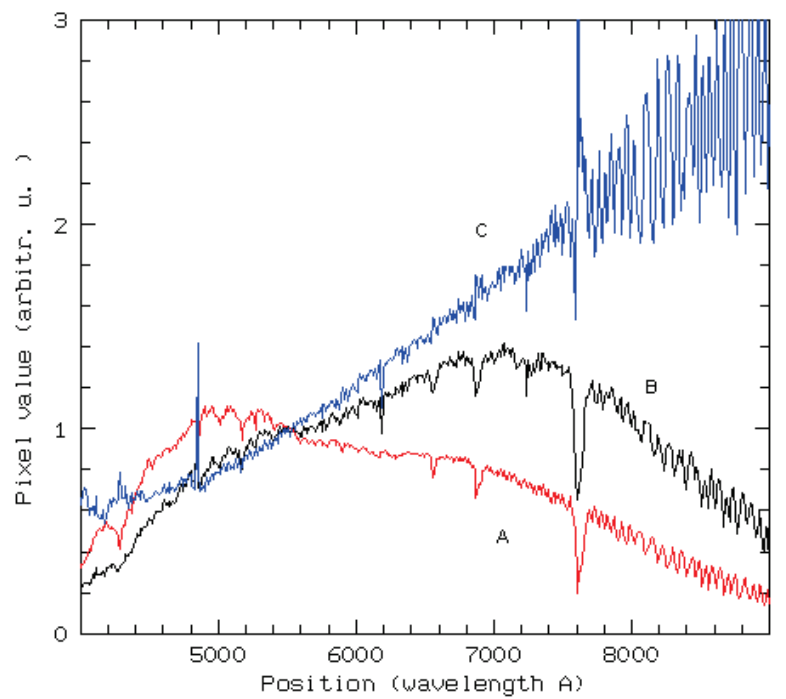

Fig. 2. The observed spectrum of the object (A) is divided by the spectrum of the solar analog (B) to obtain the reflectance spectrum (C).

\section{Determination of color indices from the measured spectra}

Some spectra measured in Tenerife were processed to extract color indices. In this operation, the calibrated reflectance spectrum, obtained in the reduction procedure mentioned above, is multiplied with a standard Sun spectrum and the color indices are calculated from the area enclosed by the color passbands. Since the spectrograph response function is not known and not enough reference stars at different air masses were measured, it was decided to compute the color indices using zeropoint magnitudes from the literature, instead of using a relative calibration based on reference stars with known indices. For the integration of the intensity in the passbands the energy is first transformed to photon count. Since the integration of energy and photon count across the same band gives slightly different results (for details see Bessell et al., 1998), the integration should be performed on the output quantity of the CCD, namely photons count.

The following steps are performed: 
- The standard Sun spectrum at the top of the atmosphere (ASTM G173-03, 2012), given in flux units [W $\mathrm{m}^{-2}$ $\mathrm{nm}^{-1}$ ], is transformed into photon units [photons s $\mathrm{m}^{-1} \mathrm{~nm}^{-1}$. Here and in the following transformations the spectra are divided in bins of $1 \mathrm{~nm}$ size and the operations are applied to each bin.

- The object reflectance spectrum is multiplied with the standard Sun spectrum.

- The resulting spectrum is multiplied with the different color passbands. The Bessel approximations (Bessell, 1990) of the classic Johnson-Cousins passbands are employed.

- The area of the spectrum covered by each color band is integrated.

- The resulting integrated value is divided by the area of the corresponding band to obtain the number of photons per unit wavelength at the band effective wavelength $\lambda_{\text {eff. }}$ The values of $\lambda_{\text {eff }}$ are taken from Bessell et al. (1998).

- The number of photons is converted to spectral flux density at the corresponding $\lambda_{\text {eff. }}$.

- The color indices are calculated from the resulting flux densities using the zeropoint magnitudes in Bessell et al. (1998).

Different factors affect the accuracy in the computation of the color indices:

- Statistics of the photon count

- Exposure artefacts: fringes, cosmics

- Errors in the calculation of the reflectance: subtraction of background, calibration of the wavelength scale, extinction correction

- Errors in the determination of the color magnitudes: conversion using zeropoint magnitudes

As can be seen from Fig. 2 the measurement noise is very low. The fringes visible as oscillations, more pronounced at higher wavelengths, will disappear after the smoothing step, but the shape of the spectrum will not essentially change. The binning step strongly improves the SNR of the measurements reducing the effect of cosmics and ensuring a clear separation of the background signal. The calibration of the wavelength scale can be performed very accurately owing to standard spectral lines: so the small calibration error is negligible. The overall error due to the latter factors does not exceed a few percent of the resulting reflectance value. The error in the zeropoint magnitudes taken from the literature, due to the observational systematic errors of the standards and comparison stars, is usually in the order of $0.02-0.03 \mathrm{mag}$, but this is not the most relevant error contribution.

From the experience gained during the observation campaigns and as mentioned in the literature (Wagner, 1992) the extinction correction is the principal source of error, due to the uncertainty in the mean extinction law. In those cases where the extinction was calculated from night measurements the error can be determined directly from the fitting procedure. Alternatively, the error using the mean extinction coefficients can be calculated from the correlation values of the extinctions in the bands B, V, R, and I, indicated in (Kidger et al., 2003). The correlation in the extinctions is due to the common influence of the amount/size of aerosol and dust present, variable during the night or period of the year, which affect the extinction in the different color regions but with different strength. The error in the color indices is then proportional to the difference of the two extinction values $\mathrm{E}_{\text {band }}$ in the respective bands, e.g. for $B-V$ the error is given by $\left(E_{B}-E_{V}\right)$ multiplied by the air mass. However, this estimate bases on the observed statistics and is not representative for all the observation nights. Special atmospheric conditions might occur on single nights and increase the extinction to extreme values, e.g. like an extinction of 0.5 mag in V referred in (Kidger et al., 2003). Also, the extinction can vary during the night or even within few hours. This would affect the measurements of the solar analogs which were taken at different times during the night. Thus the error estimate in the worst case was done using the upper and lower quartiles for the different bands indicated in (Kidger et al., 2003).

\section{RESULTS}

\section{Spectra of geostationary satellites}

Some active GEO satellites with approximately known shape and material have been observed. The knowledge of the satellite structure is important to investigate the possible identification of spacecraft surface materials from spectral data. Also satellites represent good targets to observe, since they are relative bright, usually brighter than 15 mag, and their orbits are quite accurately known. For the observations the following satellites were selected: 
Artemis (Cospar ID 01029A), Astra 1D (94070A), Meteosat MSG-1 (02040B), MSG-2 (05049B), and STTWT2 (84035A) (see Table 1).

\begin{tabular}{|c|c|c|c|c|}
\hline Satellite & Cospar ID & Type & Vis. Mag & Orbit \\
\hline Artemis & 01029A & box-wing & $10-15$ & GEO \\
\hline Astra 1D & $94070 \mathrm{~A}$ & box-wing & $10-15$ & GEO \\
\hline Meteosat MSG-1 & 02040B & cylinder & $10-15$ & GEO \\
\hline Meteosat MSG-2 & 05049B & cylinder & $10-15$ & GEO \\
\hline STTW-T2 & 84035A & cylinder & $10-15$ & GEO \\
\hline
\end{tabular}

Table 1: List of observed satellites with corresponding Cospar ID, visual magnitude, orbit, and type.

Fig. 3 shows one measured spectrum of MSG-1 and the smoothed spectrum in red. In the rest of the article smoothed spectra will be shown in those situations in which the spectrum is strongly affected by noise and fringes. In this figure and in the following the errors $\sigma_{450}$ at $450 \mathrm{~nm}$ and $\sigma_{800}$ at $800 \mathrm{~nm}$ are indicated in the figure caption. If the diagram contains several spectra error ranges are given, where higher errors correspond to higher spectral values.

The MSG satellites are spin-stabilized, rotating with more than one revolution per second, with the spin axis oriented approximately in the same direction as the Earth's axis. The surface of the cylindrical satellite mostly consists of solar cells. Observations over several seconds result in an average signal of the largest visible part of the satellite, namely its cylindrical surface. Changing the observation geometry implies different phase angles, which causes a variation in the total reflected intensity according to the amount of illuminated surface but not in the reflectance spectrum. Thus the measured spectra are not expected to change with different positions of the satellite relative to the observing station.

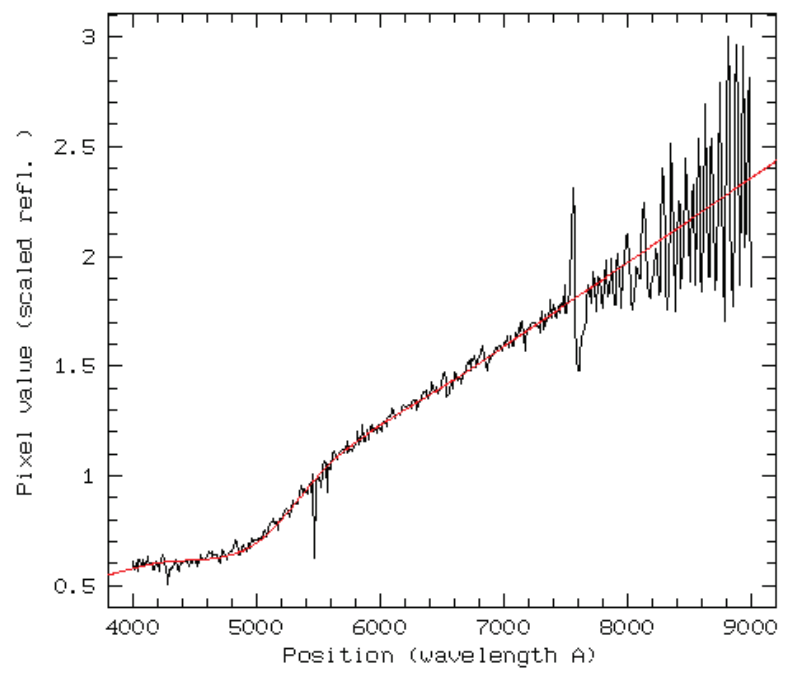

Fig. 3. Spectrum of Meteosat satellite MSG-1 (02040B). Errors: $\sigma_{450}=0.05, \sigma_{800}=0.11$.

Spectra of MSG-1 were taken with phase angles of $53^{\circ}, 27^{\circ}, 60^{\circ}$, and are shown in Fig. 4 . No distinct dependence from the phase angle is visible. The slight differences of the reflectance may be due to the not completely correct assumption of the cylindrical model for the satellite.

Conversely, the Artemis and Astra 1D satellites are three-axis stabilized and the configuration of the solar panels always points to the Sun. If we assume a homogenous diffuse scattering behavior, here a change in the spectrum with different observing angles, respectively phase angles, can be explained considering the contribution of two components of the satellite approximated by a box-wing model. The spectral signal of the wing part, or solar panels, will overlap the signal coming from the box part (main body). Depending on the reflected intensity from 
one or the other component a different spectrum may result. In Fig. 5 measurements of Artemis at $17^{\circ}$ and $52^{\circ}$ phase angle, respectively, are plotted. Contrary to expectation, no evident difference between the two spectra is visible, rather a pretty good coincidence up to about $800 \mathrm{~nm}$ can be recognized. In view of the considerations above, this result can be perhaps explained assuming that the contribution of the wings is predominant w.r.t. the one related to the box component. Another explanation could assume that one of the two contributions has a relatively flat spectrum and adding the latter does not significantly change the shape of the resulting spectrum.

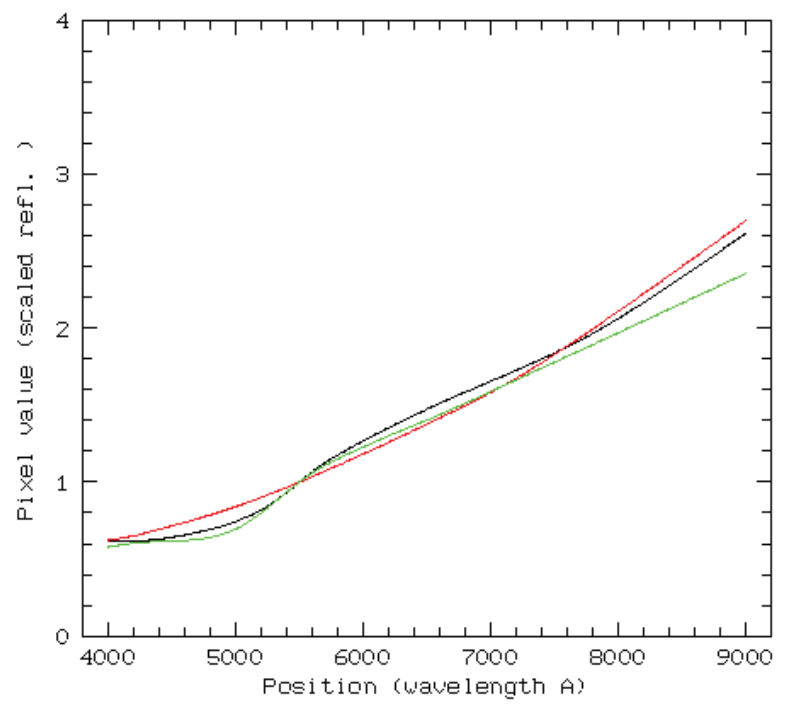

Fig. 4. Spectra of MSG-1 measured at different phase angles: $53^{\circ}$ (black), $27^{\circ}$ (red), $60^{\circ}$ (green). Errors: $\sigma_{450}=0.05, \sigma_{800}=$ 0.1 .

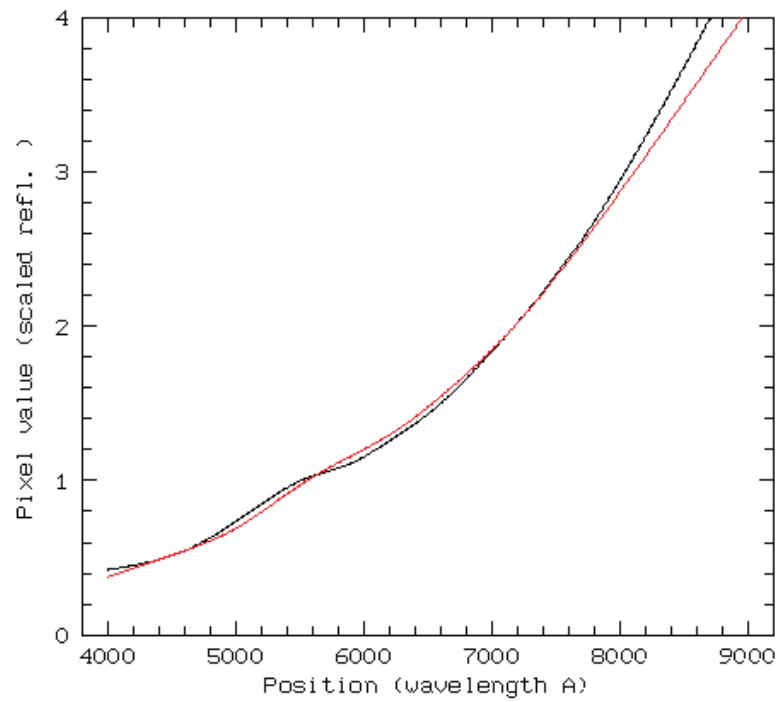

Fig. 5. Spectra of satellite Artemis at different phase angles: $17^{\circ}$ (black), $52^{\circ}$ (red). Errors: $\sigma_{450}=0.04, \sigma_{800}=0.16$.

\section{Spectra of space debris}

Several space debris objects were observed to investigate the possibility to classify them according to large-scale features of the spectra like e.g. concavity, slope, or a particular shape. Table 2 indicates the characteristics of the objects in terms of magnitude, orbit, and AMR. 
The objects are named according to the internal AIUB catalogue. This type of denomination will be used in the following text for all observed space debris objects. The magnitude errors are extracted from light curves and long term monitoring considering a one sigma deviation. The AMR value is estimated in the orbit determination procedure. GEO and GTO denote a geostationary and a geostationary transfer orbit, respectively, while eGEO indicates an 'elliptic GEO', with semimajor axis around $42000 \mathrm{~km}$ and eccentricity bigger than 0.1 .

\begin{tabular}{|c|c|c|c|}
\hline Object & Vis. Mag & Orbit & AMR $\left[\mathrm{m}^{2} / \mathrm{kg}\right]$ \\
\hline E04015A & $14.2 \pm 1.1$ & GEO & 0.2 \\
\hline E07309A & $16.4 \pm 0.9$ & GEO & 0.09 \\
\hline E08152A & $13.1 \pm 1.0$ & eGEO & 0.02 \\
\hline E08211A & $12.8 \pm 1.3$ & GEO & 0.04 \\
\hline E09231A & $12.8 \pm 0.7$ & GEO & 0.01 \\
\hline E06293A & $16.2 \pm 0.7$ & eGEO & 15.6 \\
\hline E06321D & $16.0 \pm 0.6$ & eGEO & 2.4 \\
\hline E08159C & $16.8 \pm 0.8$ & eGEO & 11.6 \\
\hline S92005 & $13.4 \pm 1.2$ & GTO & 0.02 \\
\hline S92008 & $12.7 \pm 1.5$ & GTO & 0.02 \\
\hline S95300 & $16.3 \pm 0.8$ & eGEO & 29.3 \\
\hline 84964 & $\sim 16$ & eGEO & 4.38 \\
\hline 84967 & $\sim 16$ & eGEO & 1.01 \\
\hline 84980 & $\sim 16$ & eGEO & 3.0 \\
\hline 84983 & $\sim 16$ & eGEO & 2.08 \\
\hline 84985 & $\sim 16$ & eGEO & 0.5 \\
\hline 84993 & $\sim 16$ & eGEO & 1.76 \\
\hline 84996 & $\sim 16$ & eGEO & 1.53 \\
\hline
\end{tabular}

Table 2: List of observed space debris objects with corresponding visual magnitude, orbit, and AMR.

Fig. 6 illustrates the measured spectra of the object S92005, a relative bright object with low AMR. The spectra, measured on different nights, are very similar and have pretty the same slope in the wavelength range $500 \mathrm{~nm}$ to $900 \mathrm{~nm}$. The similarity of the spectra is a confirmation of the measurement reproducibility if the observations are performed at similar phase angles and the objects have a similar attitude at the time of the acquisition. The latter cannot be always guaranteed but it is possible that the shape and material of the object are such that the measured spectrum does not depend on phase angle and attitude.

Furthermore several spectra, like the one of object S92005, exhibit an important growing reflectance in the wavelength range between $600 \mathrm{~nm}$ and $900 \mathrm{~nm}$. This behavior at longer wavelengths might be identified with the so-called 'reddening', a phenomenon associated with material surfaces in space. According to several studies, remotely observed spectra of objects in space with known surface materials turned out to be darker and redder than their corresponding laboratory spectra. It is supposed that the reddening is the result of an ageing process caused by space weathering. The structural changes at the material surface that characterize the reddening are not well understood so far. The phenomenon turned out to be difficult to model and the attempts did not give successful results (Abercromby et al., 2005). More recently the reddening effect was revisited by Bédard and Wade (2017). 


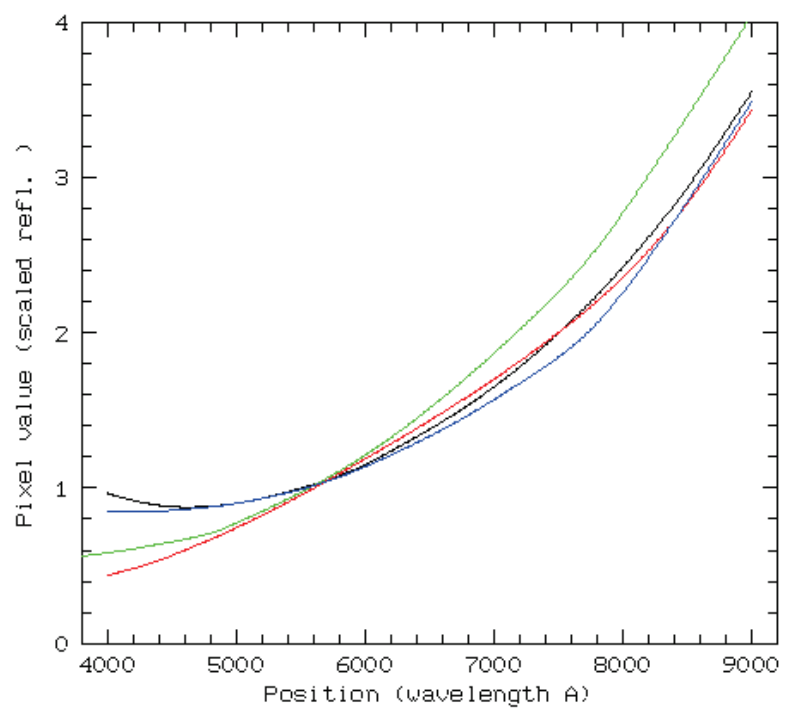

Fig. 6. Spectra of the bright GTO debris object S92005 taken on different nights (mag=13.4, AMR=0.02 $\mathrm{m}^{2} / \mathrm{kg}$ ). Errors: $\sigma_{450}$ $=0.05-0.06, \sigma_{800}=0.12-0.16$.

For some observed objects a significant different spectrum is obtained depending on the selected epoch. An example is shown in Fig. 7, where the object E08211A was observed on two different nights. Measurements on other nights showed similarities with either one or the other spectrum in Fig. 7. A possible explanation is that the object is tumbling and has two faces with different materials, so there is a certain probability of observing one or the other face.

The reflectance of object E0152A shows a different shape than in the previous spectra (Fig. 8). The shape is convex with a plateau in the region from $600 \mathrm{~nm}$ to $800 \mathrm{~nm}$. The reflectance values at $400 \mathrm{~nm}$ and $900 \mathrm{~nm}$ are similar and the object seems not to be affected by reddening.

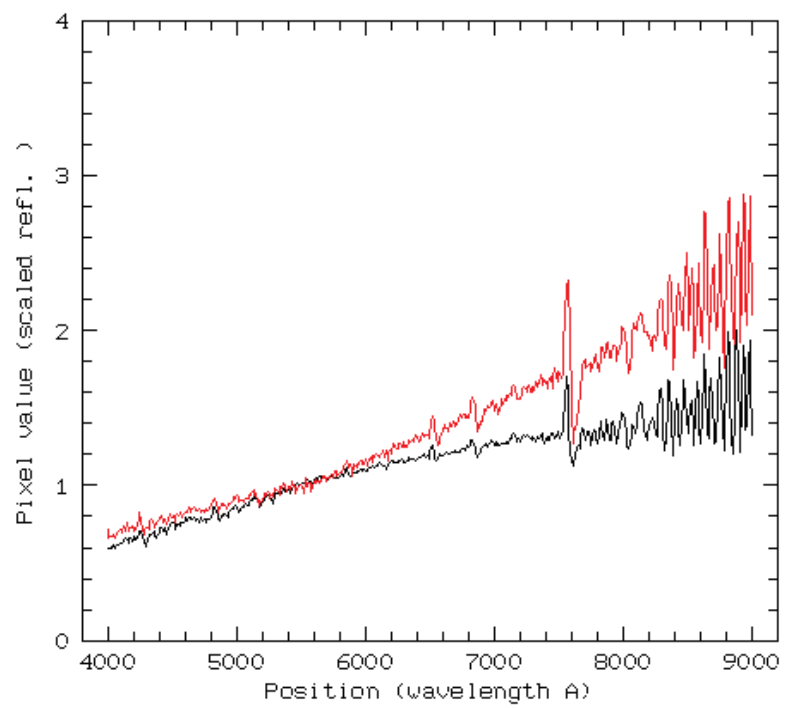

Fig. 7. Spectra of the bright GEO debris object E08211A taken on different nights (mag=12.8, AMR=0.04 $\mathrm{m}^{2} / \mathrm{kg}$ ). Errors:

$$
\sigma_{450}=0.06, \sigma_{800}=0.08-0.1 \text {. }
$$




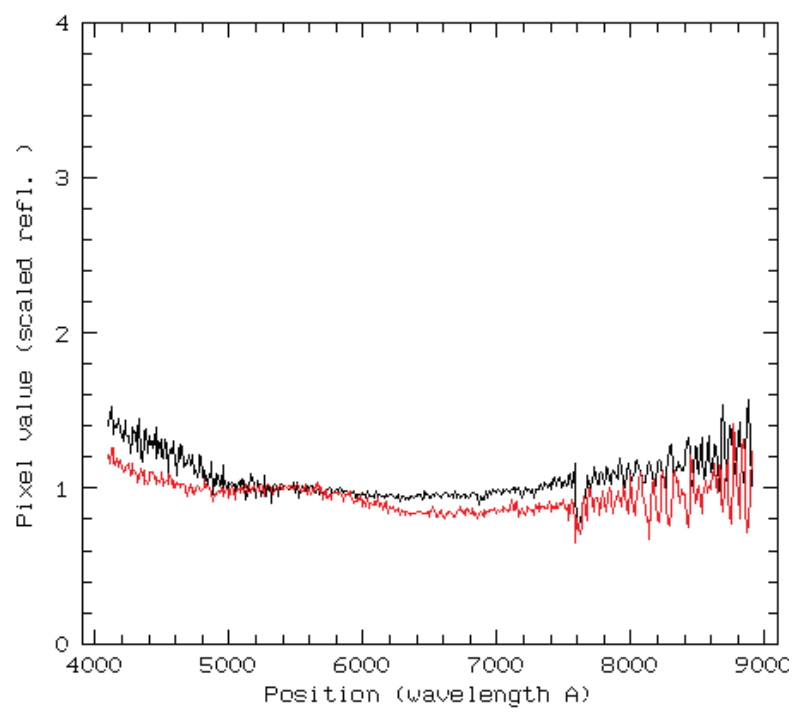

Fig. 8. Spectra of the bright 'elliptic GEO’ debris object E08152A measured on different nights (mag=13.1, AMR $=0.02 \mathrm{~m}^{2} / \mathrm{kg}$ ). Errors: $\sigma_{450}=0.08, \sigma_{800}=0.06$.

The diagrams shown until now were about low AMR objects. In the following the reflectance of some faint objects with high AMR is discussed.

The debris fragment 84985 (Fig. 9) is characterized by two clearly distinct spectra at different epochs as in the case of object E08211A. Again, this could be an indication that the object consists e.g. of different front and back side surface materials.

The spectra of the debris object 84980 (Fig. 10) exhibit a clear, sharp bend at about $500 \mathrm{~nm}$, while at higher wavelength the reflectance increases following a 'concave' trend. This 'knee' is a quite singular feature that probably characterizes the material and can be used like a sort of fingerprint. The reflectance of object S95300 with an extremely high AMR of $29.3 \mathrm{~m}^{2} / \mathrm{kg}$ is illustrated in Fig. 11. The spectral data from two nights are quite similar with a comparable positive slope (black and red plots). In contrast the data of the third night reveal an almost flat spectrum (green curve).

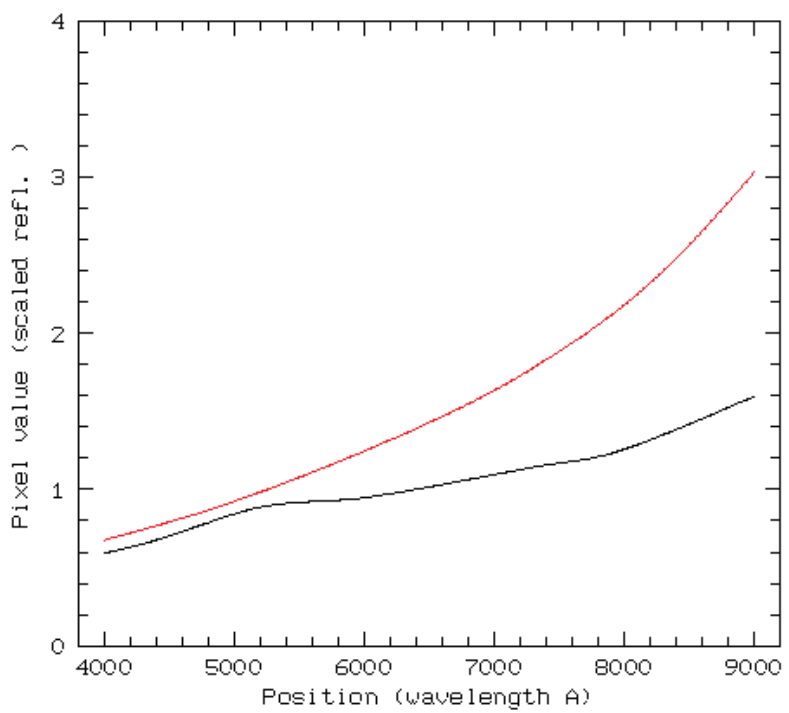

Fig. 9. Spectra of faint 'elliptic GEO’ debris object 84985 taken on different nights (mag $=\sim 16, \mathrm{AMR}=0.5 \mathrm{~m}^{2} / \mathrm{kg}$ ). Errors: $\sigma_{450}=0.06, \sigma_{800}=0.06-0.12$. 


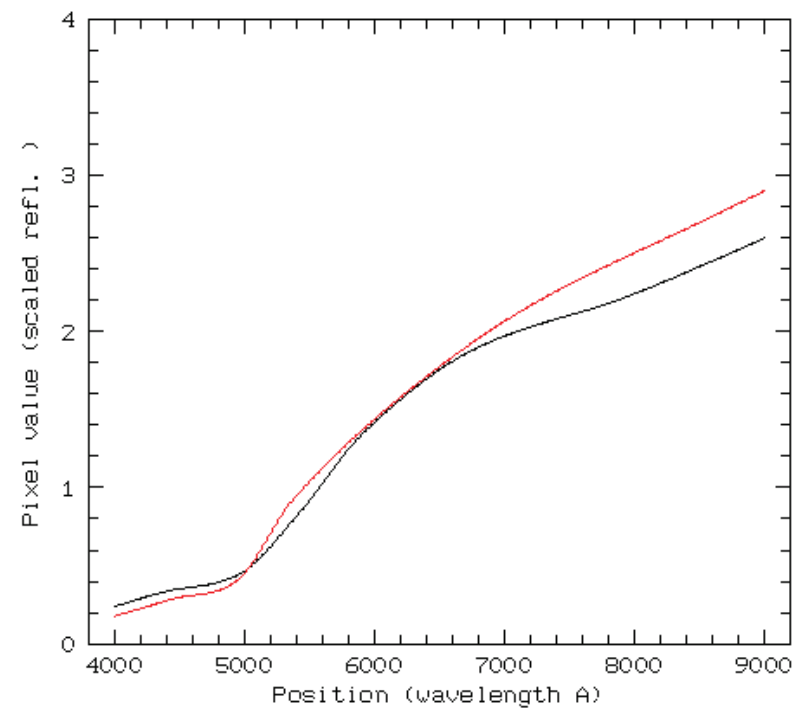

Fig. 10. Spectra of faint 'elliptic GEO' debris object 84980 measured on different nights (mag= $16, \mathrm{AMR}=3.0 \mathrm{~m} / \mathrm{kg}$ ). Errors: $\sigma_{450}=0.03, \sigma_{800}=0.12-0.14$.

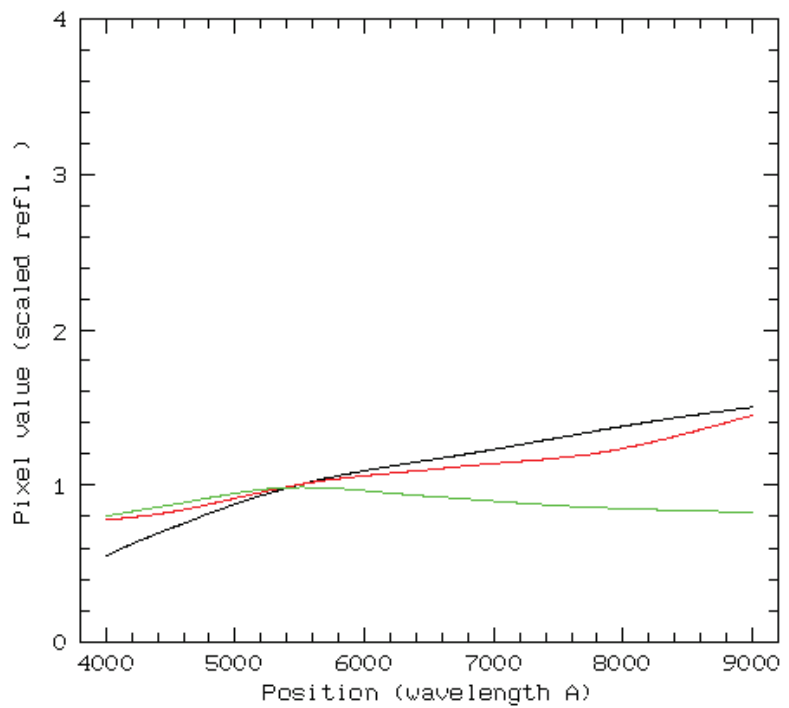

Fig. 11. Spectra of faint 'elliptic GEO' debris object $S 95300$ measured on different nights (mag=16.3, AMR=29.3 m²/kg).

Errors: $\sigma_{450}=0.06, \sigma_{800}=0.04-0.08$.

In the previous examples and in all the other spectra it is possible to find common characteristics. A preliminary attempt to classify the objects would divide the measured spectra in three categories. The first category (I) can be identified with a monotonic increasing of the reflectance with a "concave up" shape, i.e. increasing slope with increasing wavelength (Fig. 6, Fig. 9, Fig. 7 red spectrum). The quite steep slope at larger wavelength is probably related to the reddening that affects some materials. The second category (II) regroups spectra with again a monotonic increasing reflectance but with a "concave down" shape, where in this case the slope decreases with larger wavelengths (Fig. 10, Fig. 11, Fig. 7 black spectrum). A third group (III) includes spectra that are relatively flat and sometimes have a negative slope at small wavelengths in the blue range, as e.g. in Fig. 8.

\section{Comparison with laboratory spectra}

Different samples of solar cells and Multi-Layer Insulation (MLI) materials were analyzed with a spectrometer in the laboratory. Among the samples also solar cells retrieved from the Hubble Space Telescope (HST) were 
present. Two different types of MLI samples using Kapton insulation foils were available: one with a 'gold' coating, while the other with a 'silver' coating.

The remotely measured reflectance of three space objects in particular is very similar to the spectrometric measurements performed in the laboratory.

Fig. 12 displays the spectrum of the object 84980 (refer also to Fig. 10) overlapped to the 'gold' MLI spectrum measured in the laboratory. The typical knee characteristic of object 84980 is observed also in the laboratory spectrum of the 'gold' MLI. Then starting from $600 \mathrm{~nm}$ there is a slight divergence in the spectral signal, where the reflectance of the space object is higher. Perhaps this divergence can be explained with the previously mentioned reddening of space materials.

In Fig. 13 the reflectance of object S95300 (refer also to Fig. 11) is compared with the laboratory measured spectrum of the 'silver' MLI. It is remarkable that both spectra are relatively flat and follow a very similar trend, which differs only slightly at wavelengths below $500 \mathrm{~nm}$.

Both objects 84980 and S95300 have high to extremely high AMR: this may corroborate the hypothesis that these debris objects are pieces of MLI material, whose foils are very thin and light with a relatively large surface area.

In Fig. 14 the spectrum of the satellite STTW-T2 is illustrated and compared with the laboratory spectrum of a GaAs based solar cell sample. Interestingly the spectrum measured in the laboratory of the HST solar cell is similar to the one obtained from the commonly available GaAs based solar cells, which means that the prolonged exposure in the space environment did not alter its spectral characteristics. The two plots in Fig. 14 exhibit a similar trend, although the values in the blue region of the laboratory sample are significantly higher. The spectrum of STTW-T2 belongs to the category III of spectra with a negative slope in the blue range. The satellite is a spin-stabilized spacecraft with solar cells mounted on the cylindrical body. This can explain the similarity in the reflectance spectrum.

Referring to the previous three categories, the 'gold' MLI material would belong to the second category (II), while the analyzed solar cell material would be included in the third category (III). The 'silver' MLI component is relatively flat and although it does not exhibit a clear slope in the blue range, it belongs to category III.

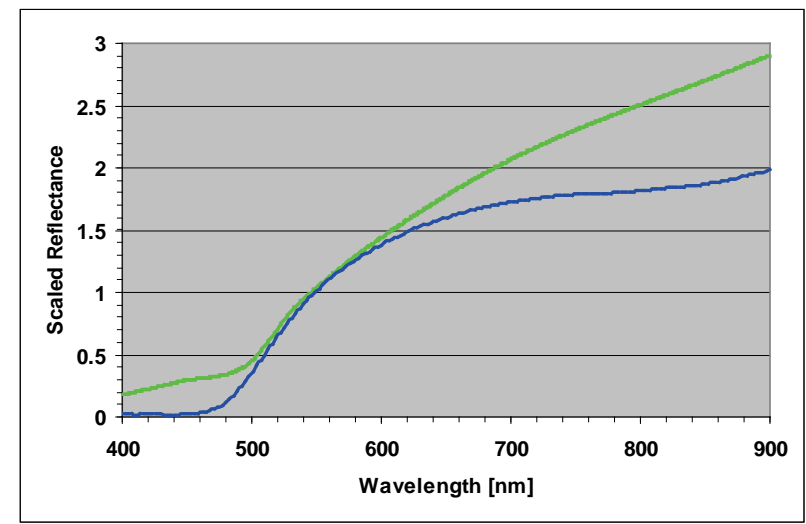

Fig. 12. Spectra of object 84980 (green; see also Fig. 10) and 'gold’ MLI (blue). 


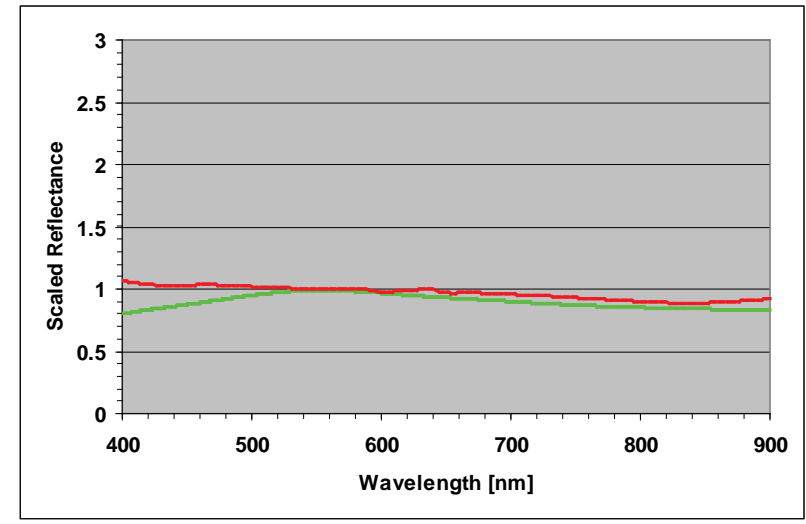

Fig. 13. Spectra of object S95300 (green; see also Fig. 11) and 'silver’ MLI (red).

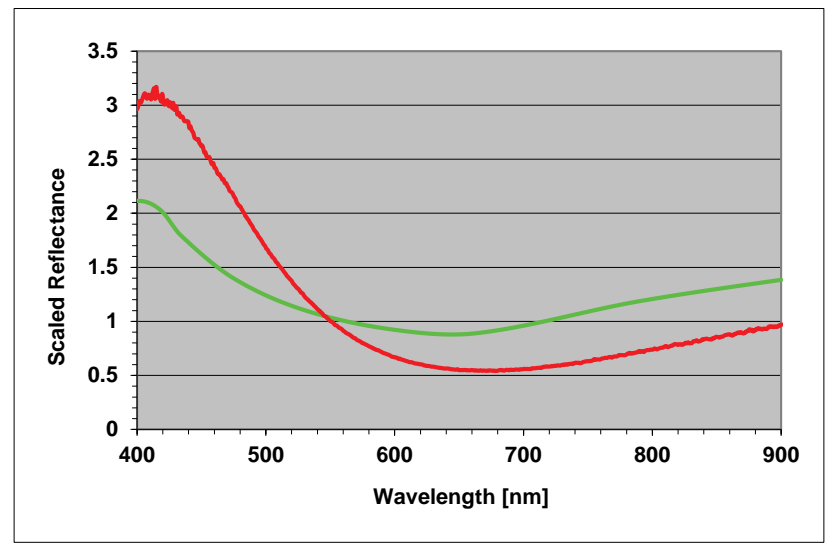

Fig. 14. Spectra of satellite STTW-T2 (green) and solar cell (red).

\section{Comparison of color indices}

Color indices were extracted from all the measured spectra of satellites and space debris objects in Table 1 and Table 2. Meteosat satellites and some high AMR objects (internal denomination starting with 849) were also measured by Cardona et al. (2016) and Rossi et al. (2012) during different observation nights. Laboratory photometric and spectroscopic average color indices of various samples were also measured by Cowardin et al. (2010). The samples include Kapton MLI material and some of the measured data were used for comparison.

In Fig. 15 and Fig. 16 the diagrams of B-V vs. V-R and B-V vs. I-R for the measured satellites are shown, respectively (see Table 3 for details). As a reference the color of the Sun is also indicated (Stritzinger et al., 2005). In blue the indices from Cardona et al. (2016) are indicated and in general are within the ranges covered by the ensemble of the observed satellites. The set of B-V indices is centered around 1, while most of the V-R and R-I values seem to be grouped around 0.7. For some satellites there is a certain color trend: for example the R-I index for Artemis (01029A) has a tendency to higher values. The satellite STTW-T2 (84035A) has a low value in all three indices. However, in general, the obtained indices of the same satellites are quite different from measurement to measurement and it is difficult to explain a specific coloration based on characteristics of the satellites. If we assume that most of the reflected light comes from solar panels we can perhaps associate the solar cell materials to the B-V region between 0.6 and 1.3, where the most objects are concentrated, which is narrower than the range covered by the space debris materials in general, as we will see later.

\begin{tabular}{|c|c|c|c|c|c|c|}
\hline Object & B-V & V-R & R-I & $\sigma_{\text {B-V }}$ & $\sigma_{\mathrm{V}-\mathrm{R}}$ & $\sigma_{\mathrm{R}-\mathrm{I}}$ \\
\hline $84035 \mathrm{~A}$ & 0.09 & 0.26 & 0.58 & 0.13 & 0.14 & 0.12 \\
\hline
\end{tabular}




\begin{tabular}{|c|c|c|c|c|c|c|}
\hline \multirow{3}{*}{ MSG2 } & 1.02 & 0.82 & 0.71 & 0.09 & 0.10 & 0.09 \\
\cline { 2 - 7 } & 0.99 & 0.73 & 0.78 & 0.10 & 0.11 & 0.09 \\
\cline { 2 - 7 } & 1.08 & 0.78 & 0.69 & 0.09 & 0.10 & 0.09 \\
\hline \multirow{3}{*}{$01029 \mathrm{~A}$} & 1.21 & 0.93 & 1.04 & 0.11 & 0.12 & 0.10 \\
\cline { 2 - 7 } & 1.20 & 0.92 & 1.06 & 0.11 & 0.12 & 0.10 \\
\hline \multirow{3}{*}{ MSG1 } & 1.17 & 0.80 & 0.87 & 0.10 & 0.11 & 0.09 \\
\cline { 2 - 7 } & 0.90 & 0.82 & 0.76 & 0.10 & 0.11 & 0.09 \\
\cline { 2 - 7 } & 0.65 & 0.45 & 0.52 & 0.10 & 0.11 & 0.09 \\
\cline { 2 - 7 } & 0.83 & 0.71 & 0.78 & 0.10 & 0.11 & 0.09 \\
\hline \multirow{3}{*}{$94070 \mathrm{~A}$} & 0.87 & 0.64 & 0.78 & 0.15 & 0.16 & 0.14 \\
\cline { 2 - 7 } & 0.90 & 0.69 & 0.83 & 0.15 & 0.16 & 0.14 \\
\cline { 2 - 7 } & 0.85 & 0.62 & 0.54 & 0.15 & 0.16 & 0.14 \\
\hline Sun & 0.65 & 0.36 & 0.35 & - & - & - \\
\hline MSG1 $^{*}$ & 0.79 & 0.64 & 0.62 & - & - & - \\
\hline MSG2 $^{*}$ & 0.60 & 0.63 & 0.60 & - & - & - \\
\hline
\end{tabular}

Table 3: List of observed satellites with respective color indices and errors $\sigma$. The Sun is given as a reference. The symbol * indicates values taken from Cardona et al. (2016).

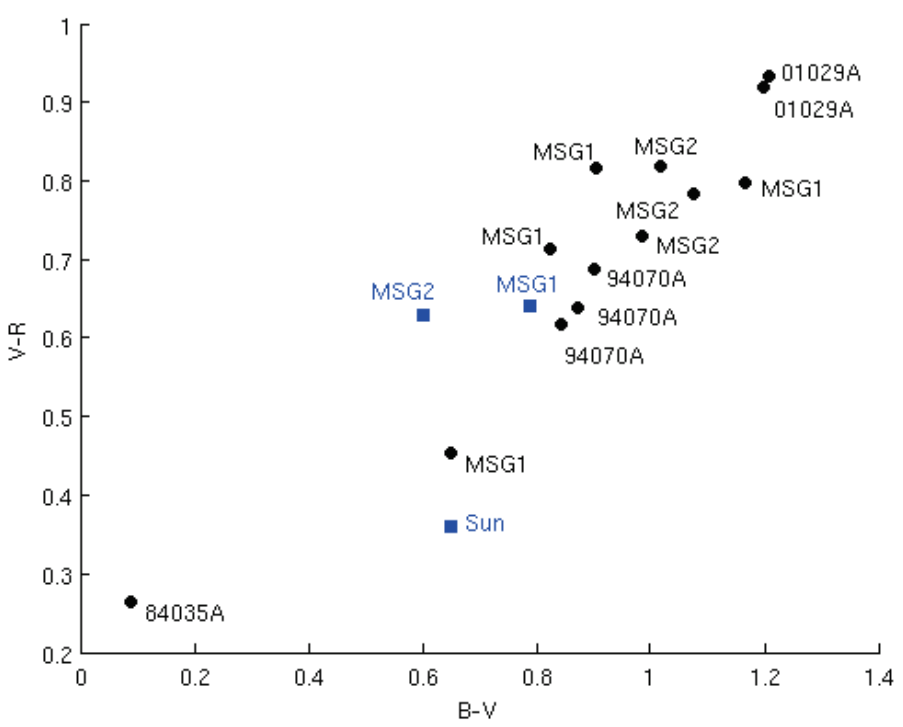

Fig. 15. Diagram B-V vs. V-R of the observed satellites. 


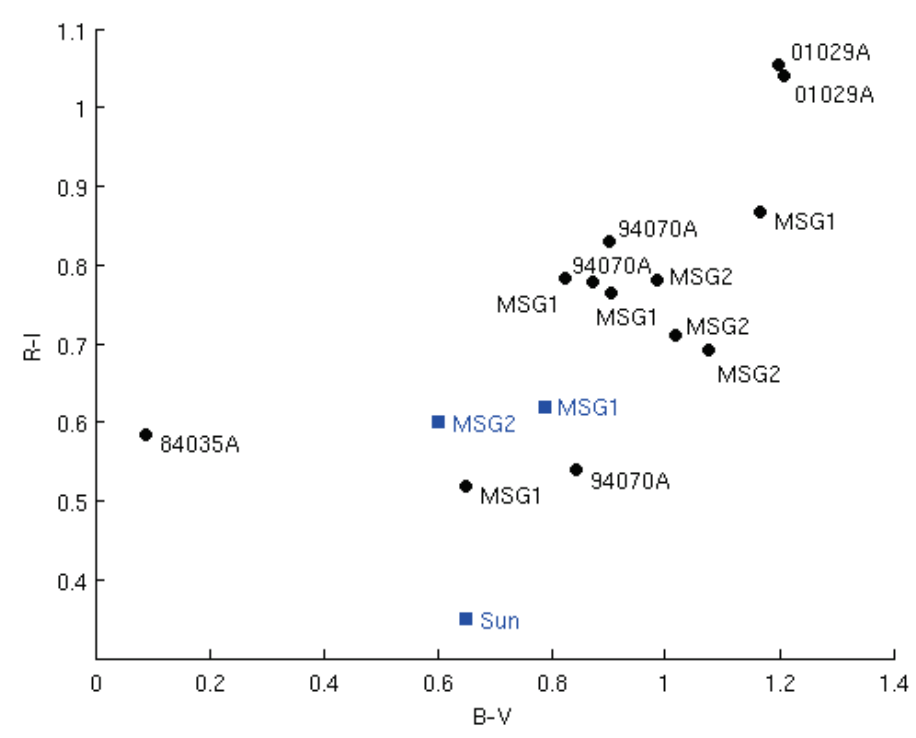

Fig. 16. Diagram B-V vs. R-I of the observed satellites.

The color indices of the observed space debris objects with AMR lower than $0.5 \mathrm{~m}^{2} / \mathrm{kg}$ are shown in Fig. 17 and Fig. 18 (see also Table 4). The diagrams illustrate the ranges covered by an inhomogeneous group of objects with a variety of different materials. Especially in the B-V index the covered range is larger than for the satellite measurements and extends from 0.4 to over 1.6. It is possible to recognize objects, like e.g. E08152A and E09231A, with particular high or low indices, but the association with a specific material is not evident. However, since the color indices are related to the shape of the spectra, they can accordingly be associated to the aforementioned empirical classification in three different categories. Higher R-I indices tend towards the category I of objects, characterized by a significant red component. On the other hand, a low B-V index could refer to category III, where a negative slope is noticed in the blue spectrum range. The partition is in particular visible if the group of high AMR objects is considered as in the next figures.

\begin{tabular}{|c|c|c|c|c|c|c|}
\hline Object & $\mathrm{B}-\mathrm{V}$ & $\mathrm{V}-\mathrm{R}$ & $\mathrm{R}-\mathrm{I}$ & $\sigma_{\mathrm{B}-\mathrm{V}}$ & $\sigma_{\mathrm{V}-\mathrm{R}}$ & $\sigma_{\mathrm{R}-\mathrm{I}}$ \\
\hline S95081 & 0.86 & 0.68 & 0.80 & 0.08 & 0.08 & 0.07 \\
\hline \multirow{3}{*}{ S92008 } & 0.64 & 0.85 & 0.89 & 0.08 & 0.09 & 0.07 \\
\cline { 2 - 7 } & 0.64 & 0.53 & 0.72 & 0.08 & 0.09 & 0.07 \\
\hline \multirow{5}{*}{ S92005 } & 0.73 & 0.77 & 0.94 & 0.08 & 0.09 & 0.08 \\
\cline { 2 - 7 } & 1.16 & 0.83 & 0.90 & 0.09 & 0.09 & 0.08 \\
\cline { 2 - 7 } & 1.04 & 0.86 & 0.96 & 0.08 & 0.09 & 0.08 \\
\cline { 2 - 7 } & 0.45 & 0.71 & 0.82 & 0.08 & 0.09 & 0.08 \\
\hline \multirow{3}{*}{ E09231A } & 1.61 & 0.99 & 0.85 & 0.12 & 0.13 & 0.11 \\
\cline { 2 - 7 } & 1.43 & 0.93 & 0.95 & 0.12 & 0.13 & 0.11 \\
\cline { 2 - 7 } & 1.62 & 0.99 & 0.86 & 0.10 & 0.10 & 0.09 \\
\cline { 2 - 7 } & 1.44 & 0.93 & 0.95 & 0.10 & 0.10 & 0.09 \\
\hline & 0.86 & 0.50 & 0.50 & 0.08 & 0.08 & 0.07 \\
\cline { 2 - 7 } & 0.86 & 0.70 & 0.74 & 0.09 & 0.09 & 0.08 \\
\cline { 2 - 7 } & 0.79 & 0.46 & 0.47 & 0.10 & 0.11 & 0.10 \\
\cline { 2 - 7 } & 1.04 & 0.66 & 0.70 & 0.10 & 0.11 & 0.09 \\
\hline \multirow{2}{*}{ E08152A } & 0.39 & 0.38 & 0.45 & 0.08 & 0.09 & 0.08 \\
\cline { 2 - 7 } & 0.54 & 0.28 & 0.41 & 0.08 & 0.09 & 0.08 \\
\hline
\end{tabular}




\begin{tabular}{|c|c|c|c|c|c|c|}
\hline \multirow{3}{*}{ E07309A } & 1.36 & 0.58 & 0.60 & 0.14 & 0.15 & 0.13 \\
\cline { 2 - 7 } & 1.10 & 0.74 & 0.70 & 0.13 & 0.14 & 0.12 \\
\cline { 2 - 7 } & 0.83 & 0.59 & 0.68 & 0.13 & 0.14 & 0.12 \\
\hline E04015A & 0.77 & 0.51 & 0.47 & 0.13 & 0.14 & 0.12 \\
\hline Sun & 0.65 & 0.36 & 0.35 & - & - & - \\
\hline
\end{tabular}

Table 4: List of observed low AMR space debris objects with respective color indices and errors $\sigma$. The Sun is given as a reference.

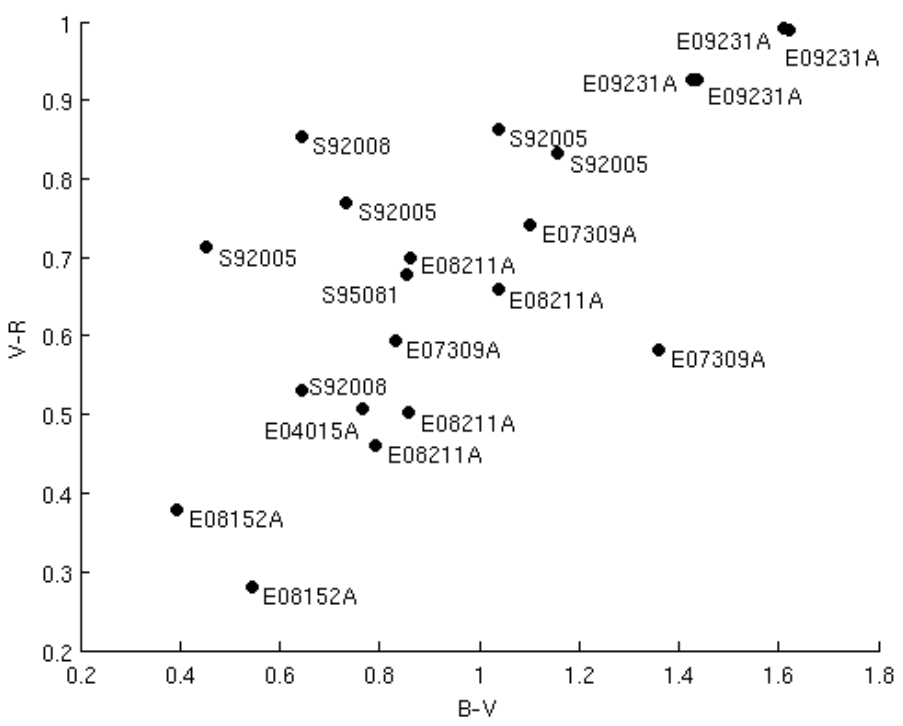

Fig. 17. Diagram B-V vs. V-R of the observed low AMR space debris objects.

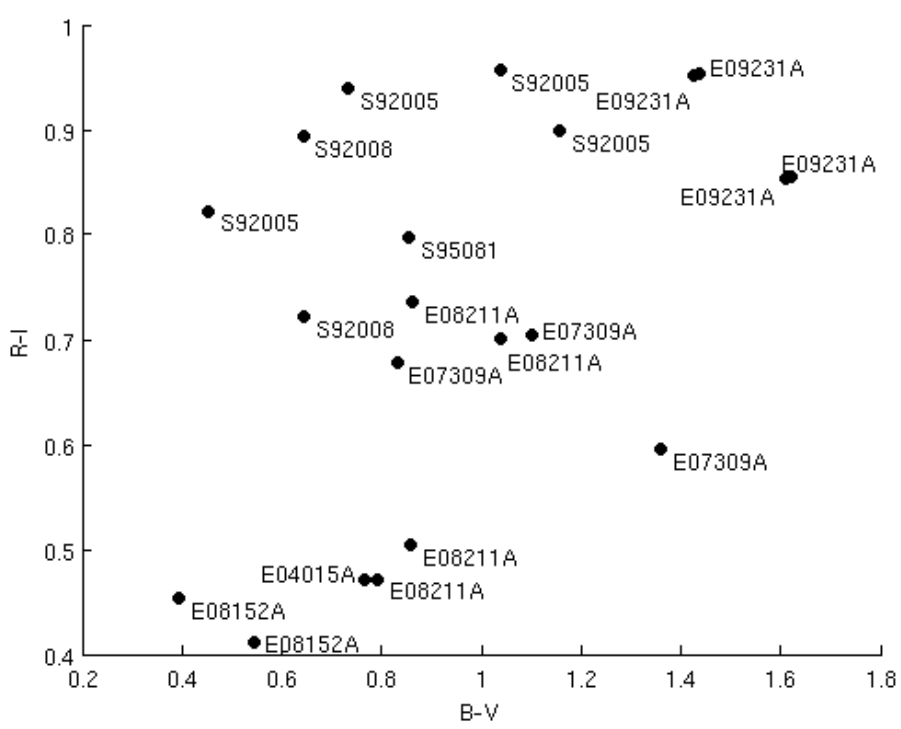

Fig. 18. Diagram B-V vs. R-I of the observed low AMR space debris objects.

Fig. 19 and Fig. 20 display the color indices of the space debris objects with high AMR larger than $0.5 \mathrm{~m}^{2} / \mathrm{kg}$ (see Table 5). In blue the measurements from Rossi et al. (2012) are indicated. While the latter measurements are quite different for object 84980 in all the indices, for object 84967 the values are closer in the indices V-R and R-I especially for one set of measured indices. 
The group of high AMR objects is a restricted group of space debris materials and part of these objects can possibly be identified with the MLI elements seen in the laboratory comparison. Already in the B-V vs. V-R diagram but more clearly in B-V vs. R-I, it is possible to identify three distinct groups of measurements (indicated with red rectangles) where according to the previous classification, the upper part would be category I, the lower right side category II, and the lower left corner category III. Also, according to the laboratory comparison, we see that object 84980 is contained in category II as expected and S95300 in category III. From the article of Cowardin et al. (2010) the spectroscopic average color indices were taken for a comparison. In that article the index B-R of the sample so-called copper MLI is 2 to 2.5 (depending on the measured side of the foil), while R-I is about 0.4. For the so-called aluminized MLI sample is $\mathrm{B}-\mathrm{R} \sim 1$ and $\mathrm{R}-\mathrm{I} \sim 0.3$. These values can be compared to the average values of our 'gold' MLI object 84980 (B-R 2.81, R-I 0.67) and 'silver' MLI object S95300 (B-R 1.3, R-I 0.48), where B-R is calculated from B-V and V-R. Although the values are not very close, the objects might be associated to the same category, respectively. It can be supposed that other objects contained in the same categories II and III belong to those families of MLI materials, while perhaps in category I thin foils of insulation or other material are for some reason affected by reddening.

\begin{tabular}{|c|c|c|c|c|c|c|}
\hline Object & $\mathrm{B}-\mathrm{V}$ & $\mathrm{V}-\mathrm{R}$ & $\mathrm{R}-\mathrm{I}$ & $\sigma_{\mathrm{B}-\mathrm{V}}$ & $\sigma_{\mathrm{V}-\mathrm{R}}$ & $\sigma_{\mathrm{R}-\mathrm{I}}$ \\
\hline \multirow{3}{*}{ S95300 } & 0.94 & 0.53 & 0.52 & 0.11 & 0.11 & 0.10 \\
\cline { 2 - 7 } & 0.82 & 0.52 & 0.48 & 0.09 & 0.10 & 0.08 \\
\cline { 2 - 7 } & 0.74 & 0.37 & 0.44 & 0.11 & 0.12 & 0.10 \\
\hline \multirow{3}{*}{ E08159C } & 0.83 & 0.52 & 0.49 & 0.10 & 0.11 & 0.10 \\
\cline { 2 - 7 } & 0.80 & 0.61 & 0.58 & 0.10 & 0.11 & 0.09 \\
\cline { 2 - 7 } & 0.61 & 0.15 & 0.15 & 0.10 & 0.11 & 0.09 \\
\hline E06321D & 1.36 & 0.79 & 0.55 & 0.10 & 0.11 & 0.09 \\
\hline E06293A & 0.48 & 0.31 & 0.44 & 0.17 & 0.18 & 0.16 \\
\hline \multirow{3}{*}{84996} & 1.30 & 0.62 & 0.57 & 0.13 & 0.13 & 0.12 \\
\cline { 2 - 7 } & 1.42 & 0.55 & 0.59 & 0.10 & 0.11 & 0.10 \\
\cline { 2 - 7 } & 1.11 & 0.75 & 0.85 & 0.11 & 0.12 & 0.10 \\
\hline 84993 & 1.06 & 0.65 & 0.71 & 0.11 & 0.12 & 0.10 \\
\hline \multirow{2}{*}{84985} & 0.95 & 0.54 & 0.62 & 0.11 & 0.11 & 0.10 \\
\cline { 2 - 7 } & 0.96 & 0.74 & 0.79 & 0.11 & 0.12 & 0.10 \\
\hline \multirow{2}{*}{84983} & 1.45 & 0.73 & 0.66 & 0.12 & 0.12 & 0.11 \\
\cline { 2 - 7 } & 1.28 & 0.60 & 0.47 & 0.11 & 0.11 & 0.10 \\
\hline \multirow{2}{*}{84980} & 1.92 & 0.99 & 0.69 & 0.19 & 0.20 & 0.17 \\
\cline { 2 - 7 } & 1.71 & 1.00 & 0.65 & 0.21 & 0.22 & 0.19 \\
\hline \multirow{2}{*}{84967} & 0.90 & 0.69 & 0.68 & 0.11 & 0.11 & 0.10 \\
\hline & 0.94 & 0.56 & 0.60 & 0.10 & 0.11 & 0.10 \\
\hline 84964 & 1.27 & 0.62 & 0.55 & 0.12 & 0.12 & 0.11 \\
\hline Sun & 0.65 & 0.36 & 0.35 & - & - & - \\
\hline $84967^{*}$ & 0.78 & 0.52 & 0.53 & - & - & - \\
\hline $84980^{*}$ & 1.42 & 0.78 & 0.48 & - & - & - \\
\hline
\end{tabular}

Table 5: List of observed high AMR space debris objects with respective color indices and errors $\sigma$. The Sun is given as a reference. The symbol * indicates values taken from Rossi et al. (2012). 


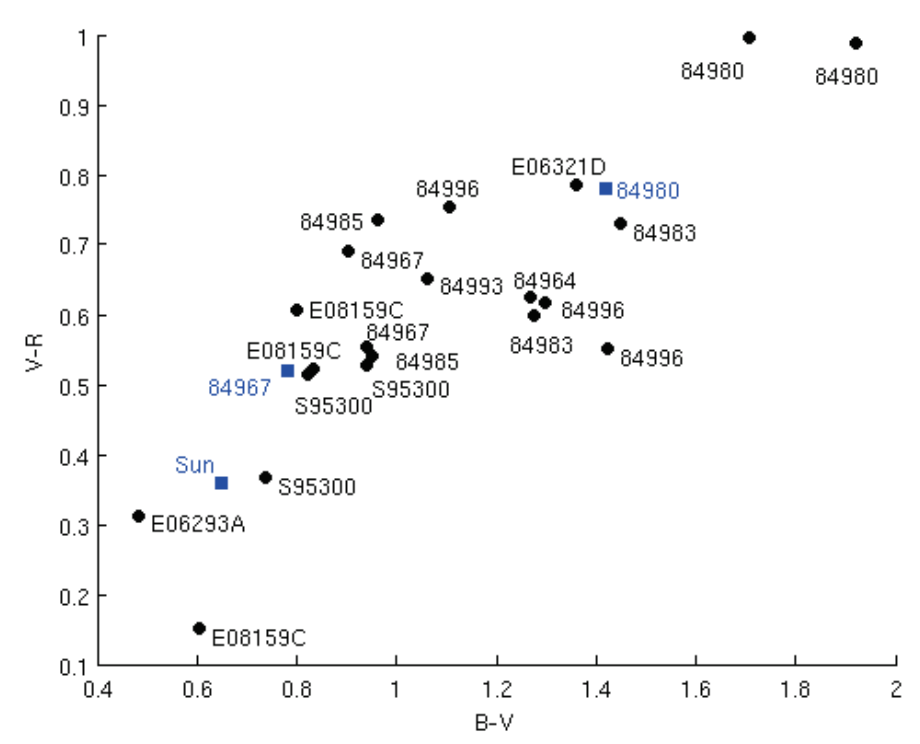

Fig. 19. Diagram B-V vs. V-R of the observed high AMR space debris objects.

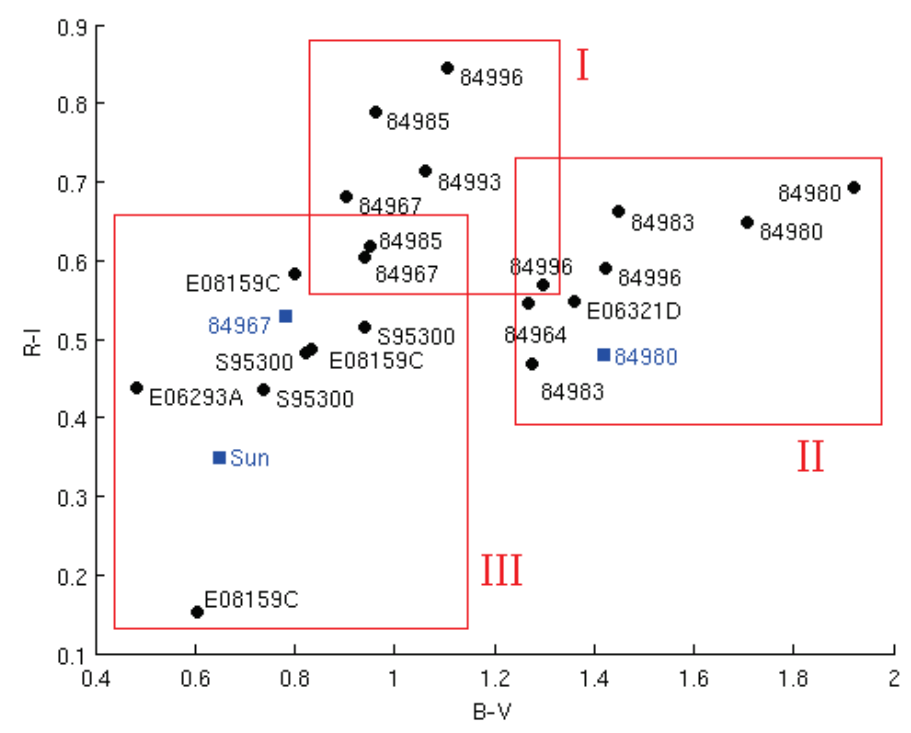

Fig. 20. Diagram B-V vs. R-I of the observed high AMR space debris objects. The red rectangles delimit the object classification: category I (upper side), category II (lower right), category III (lower left).

\section{CONCLUSIONS}

Spectroscopic observations of objects in high-altitude orbits were conducted at the ESASDT telescope in Tenerife equipped with a low-resolution spectrograph. Reflectance spectra of several satellites and space debris objects with different magnitude, AMR, and orbits were obtained.

Spin-stabilized and three-axis stabilized GEO satellites were observed at different phase angles. For the former the results are consistent with a simple geometric interpretation that assumes an average reflection of the cylindrical surface of the satellite and a spectral behavior of the surface material independent from incident and scattering angle. In the case of three-axis stabilized satellites the results indicate that, assuming a box-wings model, 
the contribution of the solar panels is predominant or that one of the two components (box or wings) has a relatively flat spectrum.

For the space debris observations a preliminary classification using three different categories purely based on the shape and appearance of the spectra was proposed:

- Category I: monotonic increasing with concave up shape

- Category II: monotonic increasing with concave down shape

- Category III: relatively flat with possible negative slope in blue range

Some of the objects which were repeatedly observed at different epochs, exhibit two different distinct spectra. This suggests that these objects have mainly two faces and, since the objects are tumbling, at different epochs one or the other face is visible during the measurement.

The effect of reddening, due to a prolonged exposure of the materials to the space environment, is not well understood. This makes the comparison of observed spectra with standard laboratory spectra difficult, as well as their interpretation. The objects in category I seem to be affected by this phenomenon.

Two debris objects with high AMR could be successfully associated with materials analyzed in the laboratory. The results show that the two debris objects are probably pieces of Kapton MLI with 'gold' and 'silver' coating, respectively. These foils composing the MLIs are light and have a relative large surface, which is consistent with high AMR values. The spectrum of a solar cell sample could be associated to the solar cells covering the cylindrical body of a satellite, under the plausible assumption that the predominant reflection comes from that part of the spacecraft.

The color indices extracted from the spectra indicate that the satellite measurements lie in a delimited B-V region, narrower than the range covered by the space debris fragments, from 0.4 to over 1.6. The comparison with color indices of the same objects in the literature does not always show a good agreement but the values lie in the same range covered by the measurements of satellites and space debris, respectively. For high AMR objects the color indices can be associated to the shape of the obtained spectra and seem to be consistent with the proposed classification in three categories. The indices of 'gold' MLI lie as expected in the category II, while 'silver' apparently belongs to category III. The B-R and R-I laboratory measurements of the latter two materials taken from the literature are comparable with the obtained results.

\section{ACKNOWLEDGEMENTS}

The ESA observations were acquired under ESA/ESOC contracts 21447/08/F/MOS. The HST solar cell sample was provided by G. Drolshagen (ESTEC). The TLEs of the objects with the denomination starting with 849 were made available by NASA in the context of an ESA/NASA joint campaign.

\section{REFERENCES}

Abercromby, K., Guyote, M., Okada, J., et al., Applying space weathering models to common spacecraft materials to predict spectral signatures, Proceedings of AMOS Technical Conference, Maui, Hawaii, USA, 2005

Abercromby, K., Okada, J., Guyote, M., et al., Comparisons of Ground Truth and Remote spectral Measurements of FORMOSAT and ANDE Spacecraft, Proceedings of AMOS Technical Conference, Maui, Hawaii, USA, 2007

ASTM G173-03(2012), Standard Tables for Reference Solar Spectral Irradiances: Direct Normal and Hemispherical on $37^{\circ}$ Tilted Surface, ASTM International, West Conshohocken, PA, 2012

Banse, K., Ponz, D., Ounnas, C., Grosbol, P., Warmels, R., The MIDAS Image Processing System, in: Instrumentation for Ground-Based Optical Astronomy, pp. 431-442, Ed. L.B. Robinson, Springer Verlag, New York, 1988

Bédard, D., Wade, G.A., Time-resolved visible/near-infrared spectrometric observations of the Galaxy 11 geostationary satellite, Advances in Space Research 59, 212-229, 2017

Bessell, M.S., UBVRI passbands, Publications of the Astronomical Society of the Pacific, vol. 102, 1181-1199, 1990

Bessell, M.S., Castelli, F., Plez, B., Model atmospheres broad-band colors, bolometric corrections and temperature calibrations for O - M stars, Astronomy and Astrophysics 333, 231-250, 1998

Cardona, T., Seitzer, P., Rossi, A., Piergentili, F., Santoni, F., BVRI photometric observations and light-curve analysis of GEO objects, Advances in Space Research 58, 514-527, 2016 
Cowardin, H., Seitzer, P., Abercromby, K., et al., Characterization of orbital debris photometric properties derived from laboratory-based measurements, Proceedings of AMOS Technical Conference, Maui, Hawaii, USA, 2010

Hardorp, J., The Sun among the stars, Astronomy and Astrophysics 63, 383-390, 1978

Jorgensen, K., Africano, J., Hamada, K., et al., Physical properties of orbital debris from spectroscopic observations, Advances in Space Research 34, 1021-1025, 2004

Kidger, M.R., Fabiola, M.-L., Narbutis, D., et al., The Atmospheric Extinction Profile in the Canary Islands: I - The visible extinction, The Observatory 123, 145-150, 2003

Landolt, A.U., UBV photoelectric sequences in the celestial equatorial selected areas 92-115, The Astronomical Journal 78 (9), 959-1021, 1973

Landolt, A.U., UBVRI Photometric standard stars in the magnitude range $11.5<\mathrm{V}<16.0$ around the celestial equator, The Astronomical Journal 104 (1), 340-491, 1992

Nishimoto, D., Africano, J., Sydney, P., et al., Spectroscopic observations of Space Objects and Phenomena using Spica and Kala at AMOS, Proceedings of SPIE, vol. 4490, San Diego, California, USA, 2001

Rossi, A., Marinoni, S., Cardona, T., et al., The Loiano campaigns for photometry and spectroscopy of geosynchronous objects, Proceedings of 63rd International Astronautical Congress, Naples, Italy, 2012

Santoni, F., Cordelli, E., Piergentili, F., Determination of disposed-upper-stage attitude motion by ground-based optical observations, Journal of Spacecraft and Rockets 50 (3), 701-708, 2013

Schildknecht, T., Optical Surveys for Space Debris, Astronomy and Astrophysics Review 14 (1), 41-111, 2007

Schildknecht, T., Musci, R., Flohrer, T., Properties of the High Area-to-Mass Ratio Space Debris Population at High Altitudes, Advances in Space Research 41, 1039-1045, 2008a

Schildknecht, T., Musci, R., Früh, C., et al., Color Photometry and Light Curve Observations of Space Debris in GEO, Proceedings of AMOS Technical Conference, Maui, Hawaii, USA, 2008b

Seitzer, P., Lederer, S.M., Cowardin, H., Cardona, T., Barker, E.S., Abercromby, K.J., Visible Light Spectroscopy of GEO Debris, Proceedings of AMOS Technical Conference, Maui, Hawaii, USA, 2012

Stritzinger, M., Suntzeff, N.B., Hamuy, M., Challis, P., Demarco, R., Germany, L., Soderberg, A.M., An atlas of spectrophotometric Landolt standard stars, Publications of the Astronomical Society of the Pacific, vol. 117, 810-822, 2005

Wagner, R.M., Point Source Spectroscopy, Astronomical CCD Observing and Reduction Techniques, ASP Conference Series, vol. 23, S. B. Howell ed., 1992

Yanagisawa, T., Kurosaki, H., Shape and motion estimate of LEO debris using light curves, Advances in Space Research 50, 136-145, 2012

Zhao, X.F., Zhang, H.Y., Yu, Y., Mao, Y.D., Multicolor photometry of geosynchronous satellites and application on feature recognition, Advances in Space Research 58, 2269-2279, 2016 